\title{
Social stimuli cause changes of plasma oxytocin and behavior in guinea pigs
}

\author{
BERNARD WALLNER ${ }^{1}$, JOHN DITTAMI ${ }^{2}$ and IVO MACHATSCHKE ${ }^{2}$
}

${ }^{1}$ Department of Anthropology, University of Vienna, Vienna, Austria.
${ }^{2}$ Department of Neurobiology and Behavioral Sciences, University of Vienna, Vienna, Austria.

\begin{abstract}
The neuropeptide oxytocin (OXT) is a key factor in the initiation and regulation of sociosexual behavior. The present study analyzes the effects of cohabitation and social challenge on plasma OXT concentration rates in guinea pig pairs in relation to male sociosexual behavior. The cohabitation phase lasted 3 days. On day 4, the pair was socially challenged by introducing an unfamiliar male. Displayed male sexual behavior varied significantly during cohabitation, with peaks on day 1 . Sociopositive behavior, i.e., side-by-side contact, was increased on days 3 and 4. Cohabitation per se led to elevated plasma OXT concentrations only in males. In contrast, both sexes reacted with increased plasma OXT concentrations to the social challenge (day 4). At that time, male OXT was significantly correlated with sexual behavior and female OXT with sociosexual behavior received from the partner. Additionally, pairs were synchronized in their OXT release during days 3 and 4. We conclude that cohabitation causes sexually dimorphic plasma OXT concentration patterns in guinea pigs. Secondly, the conformity of OXT release in both sexes may represent an endocrine marker for long-term cohabitation, which is reflected behaviorally by increased spatial proximity.
\end{abstract}

Key terms: oxytocin, behavior, guinea pig, social challenge, cohabitation.

\section{INTRODUCTION}

Social preferences and sexual contacts both seem to be key factors in regulating oxytocin (OXT) output (Carter, 1992; McCarthy, 1995). In addition, the impact of this neuropeptide on different social stimuli, such as maternal behavior (Keverne and Kendrick, 1992), pair bonds (Balaban, 2004), huddling effects (Wallner et al., 1995) or even anti-stress patterns (Gibbs, 1984; Unväs-Moberg, 1998) also is well investigated.

During lactation, both hypothalamic production sites of OXT, the parvocellular and magnocellular neurons, are stimulated transsynaptically (Crowley and Armstrong, 1992). Parvocellular neurons project into the posterior pituitary (Swanson and Sawchenko, 1983), whereas magnocellular neurons extend into limbic structures and autonomic areas of the brainstem (Buijs et al., 1983), which is proposed to coordinate OXT-mediated behavior (Witt, 1997).

Several studies documented central and/ or peripheral OXT release following sociosexual contact, as it has been shown for females after vaginocervical stimulation. (Carmichael et al., 1987; Keverne and Kendrick, 1992). In males, both sexual activity (Hughes et al., 1987; Carmichael et al., 1987; Murphy et al., 1987) and response to touch and to nociceptive stimuli instigated high OXT levels in areas of the brain as well as in the peripheral blood system (Stock, and UvnäsMoberg, 1988). There is also good evidence that OXT is actively involved in the downregulation of the hypothalamic-pituitaryadrenal axis (HPA) during stress responses (Ebner et al., 2005). This hypothesis is further supported by a study of Amico et al.

Corresponding author: Bernard Wallner, Quality Assurance Research, Department of Anthropology, University of Vienna, Maria-Theresien-Strasse 3/15a, 1090 Vienna, Austria, Tel: (43-1) 4277-18007, Fax: (43-1) 4277-9180, E-mail: bernard.wallner@univie.ac.at 
(2004), in which OXT-deficient mice displayed more anxiety-related behavior and released more corticosterone after a psychogenic stressor when compared to normal controls. The authors attributed this finding to absent anxiolytic effects of central OXT. In a previous study of ours, we observed decreased immobilization effects in relation to increased plasma OXT levels before and after the application of an abiotic stressor in paired guinea pigs (Machatschke et al., 2004).

The purpose of the present work was to analyze the effects caused by cohabitation and social challenge on plasma OXT concentration rates in male and female individuals related to male sociosexual behavior. We chose the guinea pig as a model to investigate these changes because these animals can quickly establish longlasting spatial preferences between the sexes. Furthermore, the males' behavioral and physiological response after a social challenge (confrontation with an unfamiliar male) is well documented (Sachser, 1998; Wallner and Dittami, 2003).

\section{METHODS}

\section{Animal maintenance}

Sexually experienced domestic guinea pigs from commercial sources aged one to three years were used in this study. Animals were held initially in unisexual groups in two enclosures ( $3 \mathrm{~m} \times 3 \mathrm{~m} \times 0.4 \mathrm{~m}$ ) located in the same room (room 1, $7.1 \mathrm{~m} \mathrm{x} 4.6 \mathrm{~m} \times 2.7$ $\mathrm{m})$. Photoperiod was maintained from 0700 to $1700 \mathrm{~h}$, the temperature and the humidity regulated to $20 \pm 3.5^{\circ} \mathrm{C}$ and $70 \%$, respectively. One week before the start of the experiment, all animals used in this study were placed into individual boxes $(0.30 \mathrm{~m} \times 0.50 \mathrm{~m} \times 0.20 \mathrm{~m}$ each $)$ in the same room described earlier. Pretreatment of the females lasted longer because of the need to control their reproductive state. The technique to determine the mid-phase cycle state was based on behavior and a visual check of the vaginal closure membrane (Young, 1969; Amoroso et al., 1974; Birke,
1981). Females were allowed one full estrus cycle during this pretreatment and then entered the experiment during full behavioral and vaginal estrus of their consecutive cycle.

Each animal was fed on a diet of $70 \mathrm{~g}$ fresh food and $20 \mathrm{~g}$ grain daily; water and hay were available ad libitum. Experiments were carried out in a separate room (room 2, $2.5 \mathrm{~m} \times 2.1 \mathrm{~m} \times 2.7 \mathrm{~m}$ ) located in the same laboratory suite under similar environmental conditions. All social contacts occurred between unfamiliar individuals.

Animal treatments were carried out according to the "Guiding Principles in the Care and Use of Laboratory Animals" endorsed by the American Physiological Society and by the Austrian Animal Fair Commission.

\section{Experiment}

The duration of the experiment was set for four days. On the first observation day a pair $(n=14)$ was placed into the testing arena $(1 \mathrm{~m} \times 1 \mathrm{~m})$ at $1000 \mathrm{~h}$. At this time, and on the following two days, male behavior was observed for one hour between 1000 and $1100 \mathrm{~h}$. We defined the period of the first three days as the cohabitation phase. On day four, the social challenge phase took place: an unfamiliar male was added to the pair at $1000 \mathrm{~h}$. Again, the behavior of the cohabitated male was observed for one hour. Afterwards, the intruder male was returned to a unisexual group, and the cohabited animals were put separately into their pre-experimental boxes for a further 25 hours. After each experimental run, the used arena was cleaned and disinfected. The testing behavior was recorded throughout with a ceiling-mounted video camera.

As a control group for OXT measurements, single-housed males $(n=14)$ and females $(n=14)$ were accommodated in individual boxes (room 1) during the complete test period. After each experimental run, these control animals also were re-introduced into unisexual groups.

All females, including controls, were used in vaginal estrous. 


\section{Behavioral data collection}

Males' behavior was quantified from videos using the one-zero time sampling method (Altmann, 1974; Martin and Bateson, 1993). Each observation session took 60 min and was dived into 15 -s intervals. We recorded whether a behavior pattern had occurred during the interval (see also Rhine and Linville, 1980). For recording purposes, the software program Observer 3.0 was used (Noldus, Wageningen, The Netherlands).

Behavioral definitions follow the nomenclature of Kunkel and Kunkel (1964) and Rood (1972) and consist of the following categories:

Sexual behavior: rumba-rumble (courtship encounter; slowly approaching a female, rhythmically oscillating the hindquarters from side to side and emitting a burbling vocalization; the head stretches forward, held parallel to the ground, and as the male nears the female, its body may assume a curve); naso-anal (a male lowers and turns head to sniff, lick or nuzzle the ano-genital region of a female); mounting (males rest head and foreparts on the female's back and clasp her with their forelimbs) - mounting behavior included copulation but due to the angle of the camera, copulatory success was not detectable.

Sociopositive behavior: side-by-side contact (two individuals sitting together with body contact); social grooming (two individuals sitting together may nibble each other's pelage or an animal may approach and nibble another); nose-nose (animals frequently touch noses when they meet).

\section{Blood samples and hormonal determination}

Initial blood samples were taken one day before the cohabitation period was started. Final collection was done one day after the social challenge. Blood samples from cohabitated individuals were taken at 1200 h. Immediately afterwards, individuals from the single-housed control groups were bled. Each sampling procedure lasted five minutes (including catching the animals and returning them back into the arena or their boxes) and was carried out simultaneously for males and females by two investigators. All blood samples were taken by puncturing marginal ear veins (Sachser and Pröve, 1984). Approximately $300 \mathrm{ml}$ blood was collected in heparinized capillaries. Samples were immediately put on ice. Plasma was separated by centrifugation (4000 r.p.m., $1600 \mathrm{~g}, 10 \mathrm{~min} .4^{\circ} \mathrm{C}$ ) and stored at $-30^{\circ} \mathrm{C}$ until assayed.

All samples were run in duplicate. Peripheral concentrations of OXT were determined by radioimmunoassay using the method developed by Schams et al. (1979) and Schams (1983) with ${ }^{125}$ I-marked OT as label and Rabbit no. 8 (15.1.79) antibody as antiserum (dilution 1: 100.000). Samples $(100 \mu \mathrm{l})$ were extracted on SEP-Pak C18 columns (Millipore Corp., Milford, MA, USA) with $2 \mathrm{ml}$ of tetrahydrofurane after an acetic acid wash. Recovery rates ranged from $76-78 \%$, with inter-assay variation under $20 \%$, intra-assay variation under $12 \%$, and the lowest level of detection was $0.2 \mathrm{pg}$.

\section{Statistical analyses}

A Kolmogoroff-Smirnow test confirmed a normal distribution of the data set. Behavioral data and OXT concentrations were analyzed using the General Linear Model (GLM) for repeated measures. Multiple pairwise comparisons were post hoc Bonferroni adjusted. Pairwise comparisons between cohabitated and single housed individuals were carried out with $t$ - test for independent samples. Relationships between variables were evaluated using the Pearson correlation method. Multiple correlation matrices were also Bonferroni adjusted. Values are expressed as mean $+\mathrm{SD}$; significance was accepted if $\mathrm{p}<0.05$.

\section{RESULTS}

All types of male sexual behavior showed significant variation during the observed time course (rumba-rumble: $\mathrm{p}<0.001, F=$ 9.995, df $=3$; mounting: $\mathrm{p}<0.001, \mathbf{F}=$ 9.169; df $=3$; naso-anal: $\mathrm{p}<0.001, F=$ 
18.744; df = 3) and were characterized by a significant peak on day 1 of cohabitation (Fig. 1a-c). The frequencies of the sociopositive variables nose-nose and sideby-side contact also varied significantly during the experimental phase (nose-nose: $p$ $<0.05, F=3.352$, df $=3$; side-by-side: $\mathrm{p}<$ $0.05, F=3.342, \mathrm{df}=3$ ) (Fig. 1d, e), whereas the course of social grooming behavior did not differ significantly ( $\mathrm{p}=$ $0.076, F=2.470$, df = 3) (Fig. 1f). The recorded frequencies of side-by-side contact were significantly lower on the first cohabitation day. The social challenge on day four did not affect the frequencies of male inter-sexual behavior.

Male plasma OXT concentrations were significantly elevated during the first day of cohabitation when compared to the initially measured concentrations (Fig. 2a; p < $0.010, F=3.295$, df $=5)$. A pairwise comparison between cohabited and singlehoused males was statistically significant on day $1(\mathrm{p}<0.001, t=4.208)$ and narrowly missed our significance criterion on day 4 , when the social challenge took
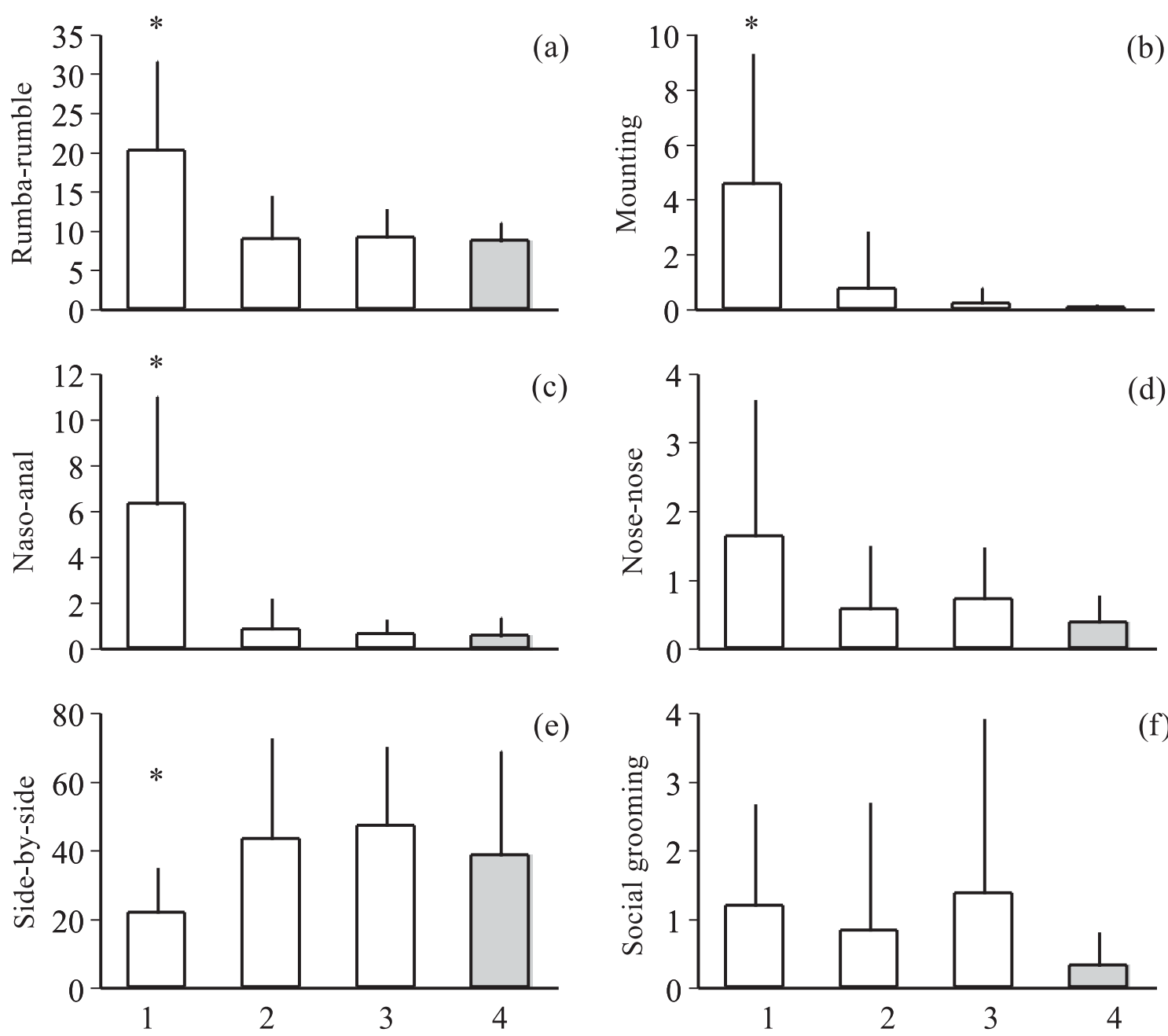

Days of cohabitation

Figure 1: Male social behavior during cohabitation (open bars) and social challenge (gray bars). GLMs are for all displayed behavior variables significant (see results) with the exception of social grooming (f). Rumba-rumble (a) and naso-anal (c) day 1 is elevated to the remaining days. Mounting (b) day 1 is increased to days 3 and 4. Frequencies of side-by-side contact are decreased at day 1 in comparison to day $3 . * \mathrm{p}<0.05$, Bonferroni corrected. Values are mean + SD. 
place $(\mathrm{p}=0.060, t=2.043)$. The remaining pairwise comparisons of the cohabitated males with single-housed males were statistically not significant (day 2: p = $0.449, t=0.782$; day $3: \mathrm{p}=0.208, t=$ 1.310). The single-housed males showed no detectable changes of their plasma OXT concentration rates during the 4-day period $(\mathrm{p}=0.621, F=0.617, \mathrm{df}=3)$.

We did not find any significant fluctuations of plasma OXT in cohabitated females (Fig. 2b). Values did not differ during the cohabitation and challenge phase when compared with before- and aftercohabitation samples $(\mathrm{p}=0.552, F=0.803$, $\mathrm{df}=5$ ). On day 4 , the social challenge phase, OXT of cohabitated females was significantly elevated in relation to singlehoused individuals ( $\mathrm{p}<0.05, t=2.424$ ). Similar to their male counterparts, singlehoused females did not show any significant changes of their plasma OXT titers during the 4-day period $(\mathrm{p}=0.304, F$ $=1.512$, $\mathrm{df}=3$ ).

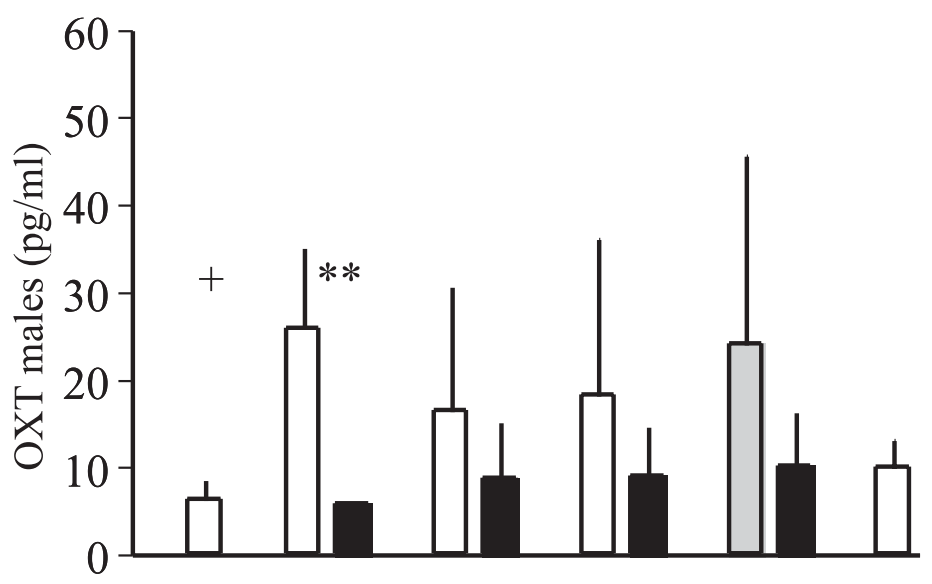

(a)

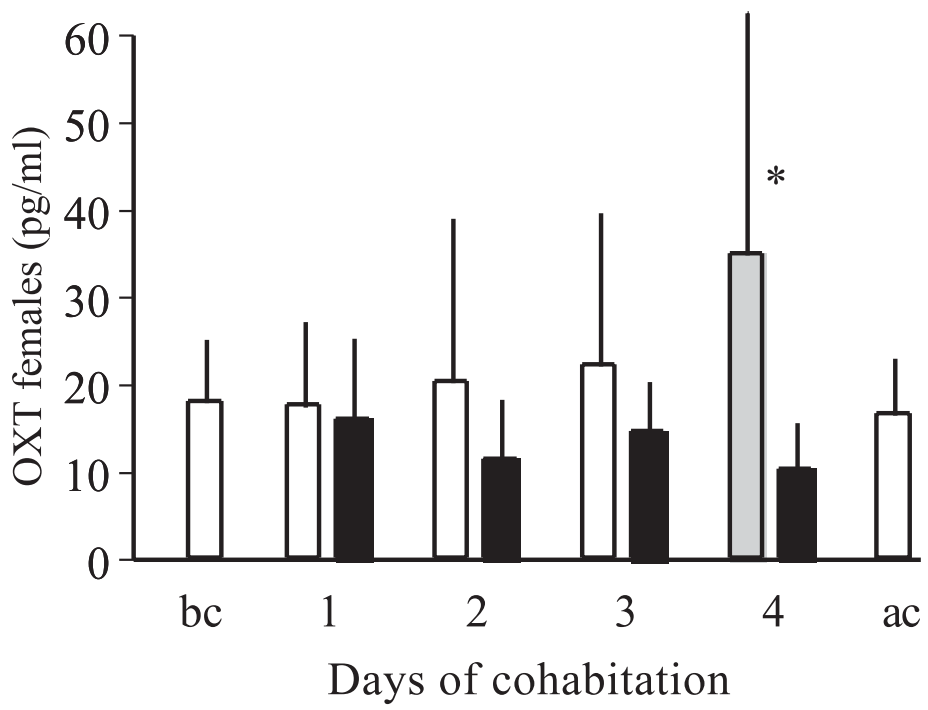

(b)

Figure 2: Open bars represent significant variation of plasma OXT concentrations in cohabitated males (a; GLM: $\mathrm{p}=0.010, F=3.295, \mathrm{df}=5$ ) and insignificant variation in cohabitated females (b; GLM: $\mathrm{p}=0.552, F=0.803, \mathrm{df}=5$ ). Black bars show plasma OXT levels of single housed individuals. In panel (a) OXT titers of day 1 are elevated to before levels $\left({ }^{+} \mathrm{p}<0.05\right.$, Bonferroni corrected) and elevated to single housed males $(* * p<0.01)$. In females (panel b) OXT titers of day 4 are different to single housed females $(* p<0.05)$. Gray bars indicate social challenge period $. \mathrm{bc}=$ before cohabitation; $\mathrm{ac}=$ after cohabitation. Values are mean $+\mathrm{SD}$. 
TABLE 1

Pearson correlation coefficients calculated between male plasma OXT concentrations and their frequencies of displayed social behavior as well as between female plasma OXT concentrations and received frequencies of male social behavior.

\begin{tabular}{|c|c|c|c|c|c|c|c|c|c|}
\hline & \multicolumn{2}{|c|}{ First } & \multicolumn{2}{|c|}{ Second } & \multicolumn{2}{|c|}{ Third } & \multicolumn{2}{|c|}{ Fourth } & \multirow{2}{*}{$\begin{array}{l}\text { day } \\
\text { OXT }\end{array}$} \\
\hline & Male & Female & Male & Female & Male & Female & Male & Female & \\
\hline \multicolumn{10}{|c|}{ Male sexual behavior } \\
\hline Rumba-rumble & -.301 & -.154 & .246 & -.444 & .188 & .075 & -.211 & .122 & \\
\hline Mounting & -.054 & -.059 & -.117 & -.174 & -.222 & -.087 & .539 & $.789 *$ & \\
\hline Naso-anal & .088 & -.359 & -.208 & -.292 & -.024 & -.156 & $.730 *$ & $.917 *$ & \\
\hline \multicolumn{10}{|c|}{ Male socio-positive behavior } \\
\hline Nose-nose & -.316 & -.357 & -.313 & .116 & .537 & .134 & .062 & .304 & \\
\hline Side-by-side & .115 & .316 & -.267 & .288 & -.298 & -.376 & -.501 & -.255 & \\
\hline Social grooming & .268 & .470 & -.025 & .092 & .261 & .673 & .067 & $.849^{*}$ & \\
\hline & & & & & & & \multicolumn{2}{|c|}{$* \mathrm{p}<0.05$} & \\
\hline
\end{tabular}

Correlation statistics of cohabitated males between their OXT levels and displayed sexual behavior showed a positive significant relationship with nasoanal during the social challenge phase (day 4 of the experiment, Table 1). All other correlations did not reach the significant criterion. Plasma OXT concentrations of cohabitated females were significantly positive correlated with sociosexual behavior received from the partner male, i.e., mounting, naso-anal, and social grooming during the challenge phase (day 4 of the experiment, Table 1). In addition, $\mathrm{OXT}$ release was significantly and positively correlated between cohabitated individuals on days 3 and 4 (Table 2).

TABLE 2

Pearson correlation coefficients calculated between secreted plasma OXT concentrations of cohabitated individuals

\begin{tabular}{ccccc}
\hline & \multicolumn{4}{c}{ Female OXT } \\
\cline { 2 - 5 } & First & Second & Third & Fourth day \\
\hline Male OXT & .561 & -.018 & $\begin{array}{r}.912 * * \\
* \mathrm{p}<0.05 ;\end{array}$ & $* * \begin{array}{c}.68 * \\
\mathrm{p}<0.01\end{array}$ \\
\hline
\end{tabular}

\section{DISCUSSION}

The results of the study show that cohabitation and the administered social challenge (introduction of an unfamiliar male to the cohabitated pair) significantly alter plasma OXT titers in guinea pigs. However, cohabitation caused a pronounced increase of plasma OXT levels only in males. This sexual dimorphism of plasma OXT concentrations during long-term cohabitation confirms results of our earlier study done on guinea pigs (Barth et al., 1997).

Generally, the release of OXT is regarded as important for initiating social interactions in both sexual and non-sexual paradigms (Williams et al., 1992, Witt et al., 1992, Haller et al., 1996). In this regard, OXT titers of guinea pig males during the first day of cohabitation seem to be triggered by the presence of females, as expressed in the increased frequency of male sociosexual behavior. However, from these results we can not conclude that OXT acts as a mediator for social behavior or contact. This finding is incidentally confirmed by missing relationships between OXT titers and the displayed behavior during the first 3 days of cohabitation. Nonetheless, we can assume that initial cohabitation leads to elevated 
plasma OXT concentrations and, consequently, to increased social behavior in guinea pig males.

Frequencies of social behavior vary between the first day and the remaining days of cohabitation (including day 4 when the social challenge took place). While male sexual behavior decreased from an initial peak, side-by-side contact as a marker of sociopositive behavior increased during the experiment. Interestingly, OXT titers of cohabitated males and females were significantly positively correlated on experimental days 3 and 4. From these results, we conclude that increased side-byside contact represents the behavioral response to long-term cohabitation among familiar individuals, as it has been shown for physical contact elsewhere (Carter et al., 1995). The strong association of plasma OXT titers between the sexes during the last two days of the experiment may represent the endocrine reaction of such a long-term cohabitation.

After the introduction of an unfamiliar male (on day 4), both cohabitated individuals reacted with increased plasma OXT levels. During that period, the frequency of male intersexual behavior was not significantly affected. However, male OXT was correlated here with sexual behavior. In addition, female OXT was related to the amount of sexual and sociopositive behaviors they received from their male partners. These results suggest that in cohabited individuals, plasma OXT concentrations are directly linked to intersexual male behavior during social challenging conditions. It is quite possible that, here, OXT function both as a cause and effect. This notion was first proposed by Uvnäs-Moberg (1997), who postulated that OXT may not only cause effects that are similar to those induced by various types of innocuous somatosensory stimulation but is also released in these situations, suggesting that it may indeed take part in the control of effects triggered by such stimuli. Regarding its functional role, our study could not clarify whether OXT is involved in a beneficial physiological reaction for cohabitated individuals, such as a down regulation of the HPA-axis (Cook, 1997) or a shift in the autonomic nervous tone from sympathetic to parasympathetic, as documented in other studies (Uvnäs-Moberg, 1997).

The behavioral and hormonal effects of confrontation experiments are well documented (Brain, 1980; Schuurman, 1980; Mazur et al., 1992; Mazur and Booth, 1998). In guinea pigs, confrontations between males were settled behaviorally after $30 \mathrm{~min}$, female-cohabited males having a significant higher probability of winning such competitions (Wallner and Dittami, 2003). Losers of such contests showed increased plasma glucucorticoid titers, indicating an elevated activity of the HPA-axes (Sachser, 1986; Sachser, 1998; Wallner and Dittami, 2003). During the present experiment, increased plasma OXT concentrations of cohabited males and females indicate a reaction that may be useful for suppressing a possible physiological stress reaction after a social challenge. A similar result was found in cohabitated guinea pigs after the application of an abiotic stressor (Machatschke et al., 2004).

In conclusion, the results of this study show that initial cohabitation causes a sexual dimorphic concentration of OXT in guinea pigs. Between the sexes, long-term cohabitation is characterized by side-byside contact as social marker and by adjusted plasma OXT release as an endocrine marker. A social challenge results in elevated plasma OXT concentrations in both sexes. The functional benefit of elevated plasma OXT titers could be interpreted as anxiolytic response to a social challenge.

\section{ACKNOWLEDGEMENTS}

The authors are grateful to D. Schams and his lab staff.

\section{REFERENCES}

ALTMANN J (1974) Observational study of behaviour: Sampling methods. Behaviour 49: 227-267

AMICO JA, MANTELLA RC, VOLLMER RR, LI X (2004) Anxiety and stress responses in female oxytocin deficient mice. J Neuroendocrinol 16: 319-324 
AMOROSO EC, DEANESLEY R, PERRY ARC (1974) The vaginal closure membrane of the guinea pig. $J$ Physiol Proceed 242: 2-3

BALABAN E (2004) Neurobiology: Why voles stick together. Nature 429: 711-712

BARTH R, WALLNER B, DITTAMI J, SCHAMS D (1997) Circulating oxytocin in male guinea pigs affected by the female cohabitation and reproductive condition. Ann NY Acad Sci 807: 593-595

BIRKE LIA (1981) Some behavioural changes associated with the guinea pig oestrous cycle. Z Tierphysiol 55: 79-89

BRAIN PF (1980) Adaptive aspects of hormonal correlates of attack and defence in laboratory mice: a study in ethobiology. Rec Progr Br Res 53: 391-413

BUIJS RM, DE VRIES GJ, VAN LEEUWEN FW, SWAAB DF (1983) Vasopressin and oxytocin: Distribution and putative functions in the brain. Prog Brain Res 60: 115-122

CARMICHAEL MS, HUMBERT R, DIXEN J, PALMISANO G, GREENLEAF W, DAVIDSON JM (1987) Plasma oxytocin increases in the human sexual response. J Clin Endocrinol Metab 64: 27-31

CARTER CS (1992) Hormonal influences on human sexual behavior. In: BECKER JB, BREEDLOVE SM, CREWS D (eds) Behavioral endocrinology. Cambridge: MIT Press. pp: 131-142

CARTER CS, DEVRIES AC, GETZ LL (1995) Physiological substrates of mammalian monogamy: The prairie vole model. Neurosci Biobehav Rev 19: 303-314

COOK CJ (1997) Oxytocin and prolactin suppress cortisol responses to acute stress in both lactating and nonlactating sheep. J Dairy Res 64: 327-39

CROWLEY WR, ARMSTRONG WE (19929 Neurochemical regulation of oxytocin secretion in lactation. Endocr Rev 13: 33-65

EBNER K, BOSCH OJ, KROMER SA, SINGEWALD N, NEUMANN ID (2005) Release of oxytocin in the rat central amygdala modulates stress-coping behavior and the release of excitatory amino acids. Neuropsychopharmacol 30: 223-230

GIBBS DM (1984) Dissociation of oxytocin, vasopressin and corticotropin secretion during different types of stress. Life Sci 35: 487-491

HALLER J, MAKARA GB, BARNA I, KOVACS K, NAGY J, VECSERNYES M (1996) Compression of the pituitary stalk elicits chronic increases in CSF vasopressin, oxytocin as well as in social investigation and aggressiveness. J Neuroendocrinol 8: 361-365

HUGHES AM, EVERITT BJ, LIGHTMAN SL, TODD K (1987) Oxytocin in the central nervous system and sexual behaviour in male rats. Brain Res 414: 133-137

KEVERNE EB, KENDRICK KM (1992) Oxytocin facilitation of maternal behavior in sheep. Ann NY Acad Sci 652: 83-101

KUNKEL P, KUNKEL I (1964) Beiträge zur Ethologie des Hausmeerschweinchen. Z Tierpsychol 21: 602-641

MACHATSCHKE I, WALLNER B, SCHAMS D, DITTAMI J (2004) Social environment affects peripheral oxytocin and cortisol during stress responses in guinea pigs. Ethology 110: 161-176

MARTIN P, BATESON P (1993) Measuring behaviour. Cambridge University Press: Cambridge

MAZUR A, BOOTH A (1998) Testosterone and dominance in men. Behav Brain Sci 21: 353-397

MAZUR A, BOOTH A, DABBS J (1992) Testosterone and chess competition. Soc Psychol Quart 55: 70-77

MCCARTHY MM (1995) Estrogen modulation of oxytocin and its relation to behavior. In: IVELL R, RUSSELL A (eds) Cellular and Molecular Approaches in Medicine and Research Oxytocin. New York: Plenum. Vol 395. pp: $235-246$

MURPHY MR, SECKL JR, BURTON S, CHECKLEY SA, LIGHTMAN SL (1987) Changes in oxytocin and vasopressin secretion during sexual activity in men. $\mathrm{J}$ Clin Endocrinol Metab 65: 738-741

RHINE RJ, LINVILLE AK (1980) Properties of one-zeroscores in observational studies of primate behaviour: The effect of assumptions on empirical analyses. Primates 21: 111-122

ROOD JP (1972) Ecological and behavioral comparisons of three genera of Argentine cavies. Anim Behav Mono 5: $1-83$

SACHSER N (1986) The effects of long-term isolation on physiology and behavior in male guinea pigs. Physiol Behav 38: 31-39

SACHSER N (1998) Of domestic and wild guinea pigs: studies in sociophysiology, domestication, and social evolution. Naturwissenschaften 85: 307-317

SACHSER N, PRÖVE E (1984) Short-term effects of residence on the testosterone responses to fighting in alpha male guinea pigs. Aggressive Behav 10: 285-292

SCHAMS D (1983) Oxytocin determination by radioimmunoassay III. Improvement to subpicogram sensitivity and application to blood levels in cyclic cattle. Acta Endocrinol 103: 180-183

SCHAMS D, SCHMIDT-POLEX B, KRUSE V (1979) Oxytocin determination by radioimmunoassay in cattle I Method and preliminary physiological data. Acta Endocrinol 92: 258-270

SCHUURMAN T (1980) Hormonal correlates of agonistic behaviour in adult male rats. Prog Brain Res 53: 415420

STOCK S, UVNÄS-MOBERG K (1988) Increased plasma levels of oxytocin in response to afferent electrical stimulation of the sciatic and vagal nerves and in response to touch and pinch in anaesthetized rats. Acta Physiol Scand 132: 29-34

SWANSON LW, SAWCHENKO PE (1983) Hypothalamic integration: Organization of the paraventricular and supraoptic nuclei. Annu Rev Neurosci 6: 269-324

UVNÄS-MOBERG K (1998) Antistress pattern induced by oxytocin. News Physiol Sci 13: 22-25

UVNÄS-MOBERG K (1997) Physiological and endocrine effects of social contact. Ann NY Acad Sci 807: 146163

WALLNER B, BARTH R, DITTAMI JP, SCHAMS D (1995) Guinea pigs (Cavia aperea f porcellus): Do they show pair-bonding behavior in relationship to oxytocin. In: IVELL R, RUSSEL G (eds) Oxytocin Cellular Approaches in Medicine and Research. New York: Plenum. Vol 395. pp: 255-257

WALLNER B, DITTAMI J (2003) Behavioural and physiological consequences of home advantage resource holding in male guinea pigs. Acta Ethol 5: 101-105

WILLIAMS JR, CARTER CS, INSEL TR (1992) Partner preference development in female prairie voles is facilitated by mating or the central infusion of oxytocin. Ann NY Acad Sci 652: 487-489

WITT DM (1997) Oxytocin-mediated sociocexual behavior. Ann NY Sci 807: 287-301

WITT DM, WINSLOW JT, INSEL TR (1992) Enhanced social interactions in rats following chronic, centrally infused oxytocin. Pharmacol Biochem Behav 43: 855861

YOUNG WC (1969) Psychobiology of sexual behavior in the guinea pig. Adv Study Behav 2: 1-110 